\title{
Follicular Fluid Perfluoroalkyl and Polyfluoroalkyl Substances Are Associated With Adverse ART Outcomes in Women With Poor Ovarian Reserve
}

\section{Yunying Cai}

Kunming University of Science and Technology https://orcid.org/0000-0003-2355-1502

LanPing Zhong

First People's Hospital of Yunnan

Ze Wu

First People's Hospital of Yunnan

Min Wang

Yunnan University

Heng Su ( $\nabla$ su_hen@hotmail.com )

First People's Hospital of Yunnan

\section{Research}

Keywords: Perfluoroalkyl and polyfluoroalkyl substances, follicular fluid, female subfertility, poor ovarian reserve

Posted Date: August 11th, 2021

DOI: https://doi.org/10.21203/rs.3.rs-789611/v1

License: (c) (1) This work is licensed under a Creative Commons Attribution 4.0 International License. Read Full License 


\section{Abstract}

\section{Background}

Previous evidence suggests that perfluoroalkyl and polyfluoroalkyl substances (PFASs) adversely affect ovarian function and female fecundity. However, the evidence remains insufficient to infer a direct relationship between PFAS exposure and adverse assisted reproductive technology (ART) outcomes. To fill this gap, we examined follicular fluid PFAS exposure and ART outcomes in patients with poor ovarian reserve (POR) in a prospective study.

Methods

In total, 147 women with POR were included. Eight PFASs were measured in follicular fluid $(n=104)$ samples using simultaneous analysis by ultra-performance liquid chromatography coupled to triple quadrupole tandem mass spectrometry. The PFAS contamination status of the patients' follicular fluid and the association between characteristics and ART outcomes were investigated by logistic regression.

Results

After adjustment for age and BMI, PFOA, PFNA, PFHxS and $\sum$ PFAS were strongly associated with a decreased probability of pregnancy (PFOA highest vs. lowest tertile: OR=1.95, 95\% Cl: 1.61, 2.38; PFNA highest vs. lowest tertile: $\mathrm{OR}=3.0,95 \% \mathrm{Cl}: 2.46,3.68$; $\mathrm{PFH} x \mathrm{~S}$ highest vs. lowest tertile: $\mathrm{OR}=1.95,95 \% \mathrm{Cl}$ : 1.61, 2.35; $\sum$ PFAS second vs. lowest tertile: OR=3.31, 95\% Cl: 2.74, 3.89). PFOS and PFUnDA were inversely associated with failed implantation. No relationships were noted between failed implantation and other PFAS analytes. The same result was obtained when using live birth as an outcome measure.

\section{Conclusions}

In women with POR, follicular fluid PFAS exposure may decrease the probability of clinical pregnancy and live birth.

\section{Introduction}

Perfluoroalkyl and polyfluoroalkyl substances (PFASs) are a class of endocrine-disrupting chemicals (EDCs) consisting of a 4-14 carbon chain decorated with several fluorine atoms and a charged functional group that have been widely used for decades in a variety of products, such as nonstick pans, water-resistant coatings and firefighting foams[1].

PFASs have recently received unparalleled attention because after years of phasing out, human biomonitoring surveys are still detecting PFASs in the majority of people worldwide. In the US National Biomonitoring Program, at least one type of PFAS was detected in the blood of nearly every person[2, 3]. 
Exposure to PFASs is known to be associated with a number of adverse health outcomes, such as the occurrence of diabetes[4], renal function[5], obesity and metabolic syndrome[6]. There is increasing evidence linking PFAS exposure to human reproductive health. Many epidemiological studies have suggested that exposure to PFASs is associated with a prolonged time to pregnancy (TTP) $[7,8,9]$.

It has been reported that poor ovarian responders (PORs) to controlled ovarian hyperstimulation ( $\mathrm{COH}$ ) have lower pregnancy rates than normal responders, and evidence has demonstrated that the live birth rate per cycle is below $10 \%[10]$. The main reason for the decreased pregnancy rates is generally associated with reduced oocyte quantity and quality. Accumulating evidence suggests that PFASs could adversely affect ovarian function by reducing ovarian hormone synthesis through activating peroxisome proliferator-activated receptors[11] and disrupting gap junction intercellular communication between oocytes and granulosa cells[12]. This evidence led us to consider whether maternal PFASs had effects on in vitro fertilization (IVF) outcomes among POR women.

Although most PFASs production is banned or restricted in developed countries[13], there has been considerable production and emission of perfluorooctanesulfonate (PFOS) and perfluorooctanoate (PFOA) in China during the last two decades[14, 15], with much of the world production shifting to China in recent years. PFASs, especially PFOS and PFOA, have been detected in infertile women in Europe and the USA. However, information on the concentrations of PFASs in subfertile women is limited in China.

To the best of our knowledge, the effect of maternal PFAS concentrations on reproductive outcomes among POR women undergoing IVF remains unexplored. In this study, we aimed to evaluate whether follicular fluid PFAS levels are a prognostic marker of adverse reproductive outcomes following IVF treatment.

\section{Methods}

\subsection{Patients}

This study was approved by the Ethics Committee of the First People's Hospital of Yunnan Province (registration number: 2017YY138). All patients signed informed consent forms before being included in the study. A total of 147 patients diagnosed with POR were enrolled. The analysis included all autologous fresh or frozen embryo transfer (FET) cycles performed from March 2017 to September 2018, with livebirths followed up to December 2019. The definition of POR was in accordance with the Bologna criteria. If at least two of the following three features are present, POR can be diagnosed: 1 ) advanced maternal age ( $\geq 40$ years) or any other risk factor for POR; 2 ) a previous POR ( $\leq 3$ oocytes with a conventional stimulation protocol); and 3) an abnormal ovarian reserve test (antral follicle count $<5-7$ follicles or anti-Müllerian hormone $<0.5-1.1 \mathrm{ng} / \mathrm{ml}$ ). Patients were excluded from the study if they had severe male-factor infertility requiring intracytoplasmic sperm injection (ICSI) or were part of a couple with genetic disorders requiring preimplantation genetic testing. Cycles where no oocytes were retrieved were excluded from the final analysis. 
Only women who achieved a clinical pregnancy rate per aspiration (CPR) or fully utilized all cryopreserved embryos during the study period were included to ensure the reliability of CPR estimates.

All women had a mild $\mathrm{COH}$ stimulation protocol: Letrozol (Furui) or clomiphene (Codal Synto) was given on day 3 of menstruation. When at least three follicles had reached $17 \mathrm{~mm}$, human chorionic gonadotrophin (hCG, 5000-10,000 IU; Serono) was injected. Oocyte retrieval was performed by the transvaginal ultrasound-guided approach 34-36 h after HCG injection. Fertilization was confirmed by the presence of two pronuclei in the zygotes.

Embryo transfers of cryopreserved embryos were performed either in an hCG-triggered natural cycle using $5000 \mathrm{IU} \mathrm{hCG}$ on the day the leading follicle was $\geq 17 \mathrm{~mm}$ or, in the case of anovulatory infertility, in estradiol- and progesterone-substituted cycles (oral estradiol $2 \mathrm{mg}$ three times daily from cycle Days 2-3 and Dydrogesterone (Abbott) added as luteal phase support 3 days prior to embryo transfer of cleavage stage frozen-thawed embryos and 6 days before transfer of vitrified-warmed blastocysts). Embryos were thawed on the transfer day. Up to two viable Day-3 embryos or one or two surviving blastocysts were transferred. In the hCG-triggered FET cycles, no luteal phase support was provided. Follicular fluid (FF) was collected only from the first puncture of one ovary. Samples were processed and stored at $-20^{\circ} \mathrm{C}$ until analysis.

\subsection{Reproductive outcomes}

The primary outcome was the CPR, defined as the presence of an intrauterine gestational sac on transvaginal ultrasound. The secondary outcomes were the cumulative live birth per aspiration (CLBR), number of oocytes retrieved and fertilization rate. CLBR was defined as at least one liveborn baby at $\geq 20$ weeks gestation resulting from an ART aspiration cycle, including all fresh and FETs resulting from the associated ovarian stimulation. Multiple deliveries from the same pregnancy were considered one live birth.

\subsection{Chemical analysis}

\subsubsection{Sample preparation and analysis}

Samples were analyzed for 8 PFASs as follows: PFOS, PFOA, heptafluorobutyric acid (PFBA), perfluorohexane sulfonate (PFHxS), perfluorononanoic acid (PFNA), perfluoroheptanoic acid (PFHpA), perfluoroundecanoic acid (PFUnDA) and perfluorodecanoic acid (PFDA), which included the two most widely studied PFASs, PFOA and PFOS, as well as those with different lengths of carbon chains (from 4 to 11 carbon atoms). Briefly, a 150- $\mu \mathrm{L}$ aliquot of each individual sample was transferred to an Eppendorf tube. After the addition of extract solution (methanol, containing isotopically labeled internal standard mixture), the samples were vortexed, centrifuged, and dried under a gentle nitrogen flow. Then, $100 \mu \mathrm{L}$ of methanol: water $=7: 3$ was added to the tube, sonicated and centrifuged, and the clear supernatant was subjected to analysis. 
UHPLC separation was carried out using an EXIONLC System (Sciex) equipped with a Waters ACQUITY UPLC BEH C18 column $(100 \times 2.1 \mathrm{~mm}, 1.7 \mu \mathrm{m}$, Waters $)$, and the injection volume was $5 \mu \mathrm{L}$. Mobile phase A was $3 \mathrm{mmol} / \mathrm{L} \mathrm{NH} 4 \mathrm{OH}$ and $3 \mathrm{mmol} / \mathrm{L}$ ammonium acetate in water, and mobile phase $B$ was acetonitrile. A SCIEX 6500 QTRAP + triple quadrupole mass spectrometer (Sciex) equipped with an IonDrive Turbo $\mathrm{V}$ electrospray ionization (ESI) interface was applied for assay development.

The MRM parameters for each of the targeted analytes were optimized using flow injection analysis by injecting the standard solutions of the individual analytes into the API source of the mass spectrometer. SCIEX Analyst Work Station Software (Version 1.6.3) and Sciex OS-Q software were employed for MRM data acquisition and processing.

\subsection{Statistical methods}

Data are given as the mean $\pm S D$, the median and intertertile range or number (\%), as appropriate. Oneway ANOVA was used to analyze continuous data with a normal distribution. Chi-squared (x2) tests were used to compare categorical data, and when the expected frequency was less than 5 , Fisher's exact test was used. Nonnormally distributed data were compared using the Wilcoxon rank-sum test. PFASs were investigated using the Spearman test. The odds ratio (OR) and 95\% confidence interval (Cl) were estimated by logistic regression. All statistical calculations were performed using SPSS 21. A $p$ value less than 0.05 was considered statistically significant.

\section{Results}

\subsection{Description of subjects}

One hundred forty-seven POR women who underwent fresh or FET cycles performed from March 2017 to September 2018 at the First People's Hospital of Yunnan Province, PR China, were enrolled. Forty-three women were excluded because their IVF cycle was cancelled, and 250 aspiration cycles undertaken by 104 women were included in the final analysis. The 104 women had a mean age of $38.6 \pm 3.2$ years, the mean CPR was $18.9 \%$, and the CLBR was $11.1 \%$. The etiological factors of females participating in ART treatment in this study were 'tubal factor' (93.7\%), endometriosis (3.1\%) and unexplained reason (3.2\%).

\subsection{PFAS concentrations in ovarian FF}

One hundred four follicular samples were analyzed for 8 PFASs. The distributions and limits of detection (LODs) of the PFAS concentrations in plasma are presented in Table 1. Seven PFASs, i.e., PFBA, PFHpA, PFOA, PFNA, PFOS, PFUnDA and PFDA, were detected in each sample. The detection frequencies of PFHxS was $98 \%$. The concentrations from highest to lowest were PFBA (median 6.63, range 0.93-31.99 $\mathrm{ng} / \mathrm{mL}$ ), PFHpA (median 1.22, range 0.02-3.73 ng/mL), PFOA (median 1.13, range 0.83-4.17 ng/mL), PFOS (median 0.73, range $0.2-9.19 \mathrm{ng} / \mathrm{mL}$ ), PFNA (median 0.51, range $0.18-1.21 \mathrm{ng} / \mathrm{mL}$ ), PFUnDA (median 0.29 , range $0.09-1.15 \mathrm{ng} / \mathrm{mL}$ ), PFDA (median 0.23 , range $0.07-0.65 \mathrm{ng} / \mathrm{mL}$ ) and PFHxS (median 0.05 , range $0.01-0.18 \mathrm{ng} / \mathrm{mL}$ ). The FF $\sum$ PFAS concentrations ranged from 2.8 to $38.1 \mathrm{ng} / \mathrm{mL}$. 
PFBA was the dominant compound in FF, with an average contribution of $54.9 \%$ $\sum$ PFAS. FF PFOS was significantly lower than that in previous studies $[16,17,18]$.

Table 1

Distribution of follicular fluid PFAS in women undergoing IVF.

\begin{tabular}{|llllll|}
\hline PFAS(ng/mL) & LOD & GM(SD) & Min & Median(IQR) & Max \\
\hline PFBA & 0.005 & $5.5(9.97)$ & 0.93 & $6.63(1.54,18.99)$ & 31.99 \\
PFHpA & 0.004 & $0.84(0.82)$ & 0.02 & $1.22(0.95,1.54)$ & 3.73 \\
PFOA & 0.005 & $1.08(0.61)$ & 0.4 & $1.13(0.83,1.41)$ & 4.17 \\
PFNA & 0.006 & $0.52(0.2)$ & 0.18 & $0.51(0.41,0.74)$ & 1.21 \\
PFHxS & 0.005 & $0.05(0.04)$ & $<$ LOD & $0.05(0.03,0.08)$ & 0.18 \\
PFOS & 0.006 & $0.86(1.37)$ & 0.2 & $0.73(0.55,1.21)$ & 9.19 \\
PFUnDA & 0.007 & $0.27(0.19)$ & 0.09 & $0.29(0.17,0.41)$ & 1.15 \\
\hline PFDA & 0.06 & $0.23(0.12)$ & 0.07 & $0.23(0.15,0.3)$ & 0.65 \\
\hline LOD, limit of detection; GM, geometric mean; SD, standard deviation; IQR, interquartile range. \\
\hline
\end{tabular}

\subsection{PFASs and ART outcome}

To test the relationship between FF PFAS contamination status and ART outcome, we first divided subjects into two groups: the pregnant group $(n=46)$ and the nonpregnant group $(n=58)$. Lower FF PFOA [0.98 $(0.82,1.21)$ vs $1.15(0.90,1.53) \mathrm{ng} / \mathrm{mL}, p=0.034]$, PFNA [0.46 $(0.38,0.67)$ vs $0.58(0.47,0.78)$ $\mathrm{ng} / \mathrm{mL}, p=0.007]$ and PFHxS $[0.04(0.03,0.06)$ vs $0.05(0.04,0.08) \mathrm{ng} / \mathrm{mL}, p=0.034]$ were found in women with ongoing pregnancy compared with women with implantation failure in the first complete cycle (Table 2). 
Table 2

Clinical parameters and PFAS levels in pregnantand non-pregnant groups after ET

\begin{tabular}{|llll|}
\hline Characteristics & Pregnant group & Non-pregnant group & $P$ \\
& $\mathrm{~N}=\mathbf{4 6}$ & $\mathrm{N}=58$ & \\
\hline Age (years) & $38(35,40)$ & $40(37.8,42)$ & 0.005 \\
\hline BMI(kg/m2) & $22.4(20.7,23.6)$ & $22.2(21.7,23.6)$ & 0.983 \\
\hline Duration of infertility(years) & $5(2,6.75)$ & $5(2.8,6)$ & 0.507 \\
\hline AMH(ng/mL) & $1.66(0.67,2.31)$ & $0.88(0.65,1.87)$ & 0.04 \\
\hline AFC & $4(3,6)$ & $3(2,5)$ & 0.015 \\
\hline Oocytes(total) & $3(2,4)$ & $3(1.8,4)$ & 0.593 \\
\hline Fertilization rate,\% & $71.4 \%$ & $54.1 \%$ & 0.018 \\
\hline PFBA(ng/mL) & $8.52(1.56,19.14)$ & $15.28(1.57,19.99)$ & 0.861 \\
\hline PFHpA(ng/mL) & $1.2(10.8,1.73)$ & $1.22(0.99,1.52)$ & 0.913 \\
\hline PFOA(ng/mL) & $0.98(0.82,1.21)$ & $1.15(0.90,1.53)$ & 0.034 \\
\hline PFNA(ng/mL) & $0.46(0.38,0.67)$ & $0.58(0.47,0.78)$ & 0.007 \\
\hline PFHxS(ng/mL) & $0.04(0.03,0.06)$ & $0.05(0.04,0.08)$ & 0.034 \\
\hline PFOS(ng/mL) & $0.87(0.63,1.26)$ & $0.72(0.57,1.19)$ & 0.88 \\
\hline PFUnDA (ng/mL) & $0.3(0.18,0.38)$ & $0.34(0.17,0.42)$ & 0.694 \\
\hline PFDA(ng/mL) & $0.24(0.16,0.29)$ & $0.24(0.17,0.39)$ & 0.304 \\
\hline$\sum$ PFAS(ng/mL) & $14.01(4.93,23.34)$ & $19.54(8.41,25.21)$ & 0.239 \\
\hline$\sum$ short-chain PFAS(ng/mL) & $10.74(2.73,20.34)$ & $16.3(4.61,21.21)$ & 0.432 \\
\hline$\sum$ long-chain PFAS(ng/mL) & $2.83(2.14,3.44)$ & $3(2.47,3.71)$ & 0.162 \\
\hline Note: Values are Mean $\pm \mathrm{SD}, \mathrm{median}$ and interquartile range and \% as indicated & \\
\hline NS nonsignificant, which means $\mathrm{P} \geq 0.05$ & & \\
\hline AFC antral follicle, BMI body $)$ & mass index, AMH anti-Mullerian hormone & \\
\hline & & & \\
\hline
\end{tabular}

Next, logistic regression was utilized to estimate the adjusted OR and $95 \% \mathrm{Cls}$. Based on previous research on PFASs[19], age and BMI were set as confounding factors and adjusted for in all of the regression models in our study. 
In the crude analysis, elevated odds of failed implantation were observed for women whose FF PFNA, PFHxS and $\sum$ PFAS were in the highest tertiles (Table 3). 
Table 3

Follicular fluid concentrations of PFASs and odds of clinical pregnancy rates

\begin{tabular}{|c|c|c|c|c|c|}
\hline $\begin{array}{l}\text { Perfluoro } \\
\text { chemicals }\end{array}$ & $\begin{array}{l}\text { Tertiles } \\
\text { (ng/mL) }\end{array}$ & $\begin{array}{l}\text { clinical pregnancy } \\
\text { rates (\%) }\end{array}$ & Cycles & $\begin{array}{l}\text { Crude OR } \\
(95 \% \mathrm{Cl})\end{array}$ & $\begin{array}{l}\text { Adjusted OR } \\
(95 \% \mathrm{Cl})\end{array}$ \\
\hline \multirow[t]{4}{*}{ PFBA } & 1st(0.93-1.59) & 30.9 & 55 & 1 (reference) & 1 (reference) \\
\hline & $\begin{array}{l}\text { 2nd }(>1.59- \\
16.73)\end{array}$ & 22.1 & 95 & $\begin{array}{l}1.58(0.75- \\
3.34)\end{array}$ & $\begin{array}{l}0.53(0.17- \\
1.68)\end{array}$ \\
\hline & $\begin{array}{l}3 r d(17.33- \\
31.99)\end{array}$ & 28 & 100 & $\begin{array}{l}1.15(0.56- \\
2.36)\end{array}$ & $\begin{array}{l}0.32(0.09- \\
1.08)\end{array}$ \\
\hline & $P$ trend & & & $P=0.449$ & $P=0.185$ \\
\hline \multirow[t]{4}{*}{ PFHpA } & 1st(0.05-1.03) & 29 & 62 & 1 (reference) & 1 (reference) \\
\hline & $\begin{array}{l}\text { 2nd }(>1.03- \\
1.32)\end{array}$ & 22.6 & 124 & $1.4(0.7-2.8)$ & $0.72(0.6-0.86)$ \\
\hline & $3 r d(1.36-3.73)$ & 31.3 & 64 & $\begin{array}{l}0.9(0.42- \\
1.93)\end{array}$ & $\begin{array}{l}0.93(0.78- \\
1.12)\end{array}$ \\
\hline & $P$ trend & & & $P=0.384$ & $P=0.117$ \\
\hline \multirow[t]{4}{*}{ PFOA } & $1 \mathrm{st}(0.4-0.92)$ & 30.8 & 91 & 1 (reference) & 1 (reference) \\
\hline & $\begin{array}{l}2 n d(>0.92- \\
1.23)\end{array}$ & 26.8 & 115 & $\begin{array}{l}1.26(0.69- \\
2.32)\end{array}$ & $0.72(0.6-0.86)$ \\
\hline & $3 r d(1.27-2.14)$ & 18.2 & 44 & $2(0.83-4.85)$ & $\begin{array}{l}1.95(1.61- \\
2.38)\end{array}$ \\
\hline & $P$ trend & & & $P=0.303$ & $P<0.001^{*}$ \\
\hline \multirow[t]{4}{*}{ PFNA } & 1st(0.18-0.45) & 40 & 80 & 1 (reference) & 1 (reference) \\
\hline & $\begin{array}{l}\text { 2nd }(>0.45- \\
0.68)\end{array}$ & 24.1 & 116 & $\begin{array}{l}1.93(1.05- \\
3.57)\end{array}$ & $\begin{array}{l}1.15(0.96- \\
1.34)\end{array}$ \\
\hline & $3 r d(0.71-1.21)$ & 11.1 & 54 & $\begin{array}{l}4.92(1.89- \\
12.8)\end{array}$ & $3(2.46-3.68)$ \\
\hline & $P$ trend & & & $P=0.003^{*}$ & $P<0.001^{*}$ \\
\hline \multirow[t]{2}{*}{ PFHxS } & $1 \mathrm{st}(0.01-0.04)$ & 37.1 & 89 & 1 (reference) & 1 (reference) \\
\hline & $\begin{array}{l}\text { 2nd }(>0.04- \\
0.08)\end{array}$ & 27.5 & 91 & $\begin{array}{l}1.56(0.83- \\
2.92)\end{array}$ & $0.72(0.6-0.86)$ \\
\hline
\end{tabular}

$\mathrm{OR}$, odds ratio; $\mathrm{Cl}$, confidence interval

P-value for test of trend across tertiles

Adjusted for age(continuous),BMI(continuous) 


\begin{tabular}{|c|c|c|c|c|c|}
\hline \multirow[t]{3}{*}{$\begin{array}{l}\text { Perfluoro } \\
\text { chemicals }\end{array}$} & $\begin{array}{l}\text { Tertiles } \\
\text { (ng/mL) }\end{array}$ & $\begin{array}{l}\text { clinical pregnancy } \\
\text { rates (\%) }\end{array}$ & Cycles & $\begin{array}{l}\text { Crude OR } \\
(95 \% \mathrm{Cl})\end{array}$ & $\begin{array}{l}\text { Adjusted OR } \\
(95 \% \mathrm{Cl})\end{array}$ \\
\hline & $\begin{array}{l}3 r d(>0.08- \\
0.18)\end{array}$ & 11.4 & 70 & $\begin{array}{l}4.57(1.95- \\
10.71\end{array}$ & $\begin{array}{l}1.95(1.61- \\
2.35)\end{array}$ \\
\hline & $P$ trend & & & $P=0.002$ & $P<0.001^{\star}$ \\
\hline \multirow[t]{4}{*}{ PFOS } & $1 \mathrm{st}(0.34-0.69)$ & 20 & 80 & 1 (reference) & 1 (reference) \\
\hline & $\begin{array}{l}\text { 2nd }(0.69- \\
1.21)\end{array}$ & 23.4 & 94 & $\begin{array}{l}0.82(0.39- \\
1.69)\end{array}$ & $0.4(0.23-0.49)$ \\
\hline & $3 r d(1.21-9.19)$ & 36.8 & 76 & $\begin{array}{l}0.43(0.21- \\
0.88)\end{array}$ & $\begin{array}{l}0.53(0.44- \\
0.64)\end{array}$ \\
\hline & $P$ trend & & & $P=0.044^{*}$ & $P=0.013$ \\
\hline \multirow[t]{4}{*}{ PFUnDA } & $1 \mathrm{st}(0.09-0.22)$ & 15.9 & 88 & 1 (reference) & 1 (reference) \\
\hline & $\begin{array}{l}\text { 2nd }(>0.22- \\
0.39)\end{array}$ & 44.9 & 78 & $\begin{array}{l}0.23(0.1- \\
0.48)\end{array}$ & $\begin{array}{l}0.17(0.15- \\
0.21)\end{array}$ \\
\hline & $\begin{array}{l}3 r d(>0.39- \\
1.15)\end{array}$ & 28.6 & 84 & $\begin{array}{l}0.47(0.23- \\
0.99)\end{array}$ & $\begin{array}{l}0.35(0.28- \\
0.42)\end{array}$ \\
\hline & $P$ trend & & & $P<0.001^{\star}$ & $P<0.001$ \\
\hline \multirow[t]{4}{*}{ PFDA } & $1 \mathrm{st}(0.07-0.22)$ & 27.8 & 72 & 1 (reference) & 1 (reference) \\
\hline & $\begin{array}{l}2 \mathrm{nd}(>0.22- \\
0.3)\end{array}$ & 27.9 & 122 & $\begin{array}{l}0.72(0.38- \\
1.36)\end{array}$ & $\begin{array}{l}0.43(0.36- \\
0.52)\end{array}$ \\
\hline & $3 r d(0.33-0.65)$ & 21.4 & 56 & $\begin{array}{l}1.02(0.45- \\
2.29)\end{array}$ & $0.92(0.76-1.1)$ \\
\hline & Ptrend & & & $P=0.634$ & $P=0.11$ \\
\hline \multirow[t]{4}{*}{$\sum$ PFAS } & 1st(2.8-6.85) & 42.6 & 61 & 1 (reference) & 1 (reference) \\
\hline & $\begin{array}{l}\text { 2nd(>6.85- } \\
22.33)\end{array}$ & 27.9 & 111 & $\begin{array}{l}1.61(0.85- \\
3.08)\end{array}$ & $\begin{array}{l}3.31(2.74- \\
3.89)\end{array}$ \\
\hline & $\begin{array}{l}3 r d(>22.33- \\
38.08)\end{array}$ & 23.1 & 78 & $\begin{array}{l}2.48(1.19- \\
5.15)\end{array}$ & $\begin{array}{l}1.85(1.13- \\
2.22)\end{array}$ \\
\hline & $P$ trend & & & $P=0.05$ & $P<0.001$ \\
\hline \multicolumn{6}{|c|}{ OR, odds ratio; $\mathrm{Cl}$, confidence interval } \\
\hline \multicolumn{6}{|c|}{ P-value for test of trend across tertiles } \\
\hline Adjusted fol & ge(continuous), & II(continuous) & & & \\
\hline
\end{tabular}


After adjustment for age and BMI, PFOA in the highest tertile was strongly associated with an increased risk of failed implantation ( $\mathrm{OR}=1.95,95 \% \mathrm{Cl}$ : 1.61, 2.38). Moreover, the association of PFNA, PFHxS and $\sum$ PFAS with failed implantation persisted and was even stronger (PFNA highest vs. lowest tertile: OR = 3.00, 95\% Cl: 2.46, 3.68; PFHxS highest vs. lowest tertile: $\mathrm{OR}=1.95,95 \% \mathrm{Cl}: 1.61,2.35 ;$; PFAS second vs. lowest tertile: OR $=3.31,95 \% \mathrm{Cl}: 2.74,3.89$ ) (Table 3). In contrast, PFOS and PFUnDA were inversely associated with failed implantation. There were no relationships between failed implantation and other PFAS analytes.

Furthermore, when using live birth as an outcome measure, the same result was obtained. After adjustment for age and BMI, FF PFOA, PFNA, PFHxS and $\sum$ PFAS were strongly associated with increased risks of failed live birth (PFOA highest vs. lowest tertile: $\mathrm{OR}=2.04,95 \% \mathrm{Cl}: 1.63,2.56$; PFNA highest vs. lowest tertile: $\mathrm{OR}=7.03,95 \% \mathrm{Cl}: 5.35,9.25$; PFHxS highest vs. lowest tertile: $\mathrm{OR}=2.04,95 \% \mathrm{Cl}$ : 1.63, 2.56; $\sum$ PFAS second vs. lowest tertile: $\left.\mathrm{OR}=1.76,95 \% \mathrm{Cl}: 1.43,2.16\right)$ (Table 4$)$. Inverse associations were observed for the highest levels of PFOS and PFUnDA. 
Table 4

Follicular fluid concentrations of PFASs and odds of cumulative live birth per aspiration

\begin{tabular}{|c|c|c|c|c|c|}
\hline Perfluorochemicals & Tertiles(ng/mL) & $\begin{array}{l}\text { cumulative live } \\
\text { birth per } \\
\text { aspiration (\%) }\end{array}$ & Cycles & $\begin{array}{l}\text { Crude } \\
\text { OR(95\%Cl) }\end{array}$ & $\begin{array}{l}\text { Adjusted } \\
\text { OR } \\
(95 \% \mathrm{Cl})\end{array}$ \\
\hline \multirow[t]{4}{*}{ PFBA } & 1st(0.93-1.59) & 7.27 & 55 & 1 (reference) & $\begin{array}{l}1 \\
\text { (reference) }\end{array}$ \\
\hline & $\begin{array}{l}\text { 2nd }(>1.59- \\
16.73)\end{array}$ & 10.5 & 95 & $\begin{array}{l}0.67(0.19- \\
2.24)\end{array}$ & $\begin{array}{l}0.53(0.17- \\
1.68)\end{array}$ \\
\hline & $\begin{array}{l}3 r d(17.33- \\
31.99)\end{array}$ & 16 & 100 & $0.41(0.13,1.31)$ & $\begin{array}{l}0.32(0.19- \\
1.08)\end{array}$ \\
\hline & $P$ trend & & & $P=0.249$ & $P=0.185$ \\
\hline \multirow[t]{4}{*}{ PFHpA } & 1st(0.05-1.03) & 6.45 & 62 & 1 (reference) & $\begin{array}{l}1 \\
\text { (reference) }\end{array}$ \\
\hline & $\begin{array}{l}\text { 2nd }(>1.03- \\
1.32)\end{array}$ & 12.9 & 124 & $\begin{array}{l}0.47(0.15- \\
1.45)\end{array}$ & $\begin{array}{l}0.19(0.15- \\
1.03)\end{array}$ \\
\hline & $3 r d(1.36-3.73)$ & 15.6 & 64 & $0.37(0.11,1.26)$ & $\begin{array}{l}0.31(0.24- \\
1.18)\end{array}$ \\
\hline & $P$ trend & & & $P=0.276$ & $P=0.19$ \\
\hline \multirow[t]{4}{*}{ PFOA } & $1 s t(0.4-0.92)$ & 10.9 & 91 & 1 (reference) & $\begin{array}{l}1 \\
\text { (reference) }\end{array}$ \\
\hline & $\begin{array}{l}\text { 2nd }(>0.92- \\
1.23)\end{array}$ & 13.9 & 115 & $\begin{array}{l}0.53(0.33- \\
1.78)\end{array}$ & $\begin{array}{l}0.65(0.55- \\
1.56)\end{array}$ \\
\hline & $3 r d(1.27-2.14)$ & 9.09 & 44 & $\begin{array}{l}1.24(0.37- \\
4.18)\end{array}$ & $\begin{array}{l}2.04(1.63- \\
2.56)\end{array}$ \\
\hline & $P$ trend & & & $P=0.66$ & $P<0.001^{*}$ \\
\hline \multirow[t]{4}{*}{ PFNA } & 1st(0.18-0.45) & 17.5 & 80 & 1 (reference) & $\begin{array}{l}1 \\
\text { (reference) }\end{array}$ \\
\hline & $\begin{array}{l}\text { 2nd }(>0.45- \\
0.68)\end{array}$ & 12.1 & 116 & $\begin{array}{l}1.45(0.65- \\
3.25)\end{array}$ & $\begin{array}{l}1.04(0.87- \\
1.25)\end{array}$ \\
\hline & $3 \operatorname{rd}(0.71-1.21)$ & 3.7 & 54 & $\begin{array}{l}5.2(1.13- \\
23.88)\end{array}$ & $\begin{array}{l}7.03(5.35- \\
9.25)\end{array}$ \\
\hline & $P$ trend & & & $P=0.09$ & $P<0.001^{*}$ \\
\hline
\end{tabular}

OR, odds ratio; $\mathrm{Cl}$, confidence interval

P-value for test of trend across tertiles

Adjusted for age(continuous),BMI(continuous) 


\begin{tabular}{|c|c|c|c|c|c|}
\hline Perfluorochemicals & Tertiles(ng/mL) & $\begin{array}{l}\text { cumulative live } \\
\text { birth per } \\
\text { aspiration (\%) }\end{array}$ & Cycles & $\begin{array}{l}\text { Crude } \\
\text { OR(95\%Cl) }\end{array}$ & $\begin{array}{l}\text { Adjusted } \\
\text { OR } \\
(95 \% \mathrm{Cl})\end{array}$ \\
\hline \multirow[t]{4}{*}{ PFHxS } & $1 \mathrm{st}(0.01-0.04)$ & 14.6 & 89 & 1 (reference) & $\begin{array}{l}1 \\
\text { (reference) }\end{array}$ \\
\hline & $\begin{array}{l}\text { 2nd }(>0.04- \\
0.08)\end{array}$ & 14.9 & 91 & $\begin{array}{l}1.03(0.45- \\
2.36)\end{array}$ & $\begin{array}{l}0.65(0.55- \\
1.77)\end{array}$ \\
\hline & $\begin{array}{l}3 \operatorname{rd}(>0.08- \\
0.18)\end{array}$ & 5.71 & 70 & $\begin{array}{l}2.82(0.89- \\
9.08)\end{array}$ & $\begin{array}{l}2.04(1.63- \\
2.56)\end{array}$ \\
\hline & $P$ trend & & & $P=0.184$ & $P<0.001^{*}$ \\
\hline \multirow[t]{4}{*}{ PFOS } & 1st(0.34-0.69) & 2.5 & 80 & 1 (reference) & $\begin{array}{l}1 \\
\text { (reference) }\end{array}$ \\
\hline & $\begin{array}{l}\text { 2nd(0.69- } \\
1.21)\end{array}$ & 14.9 & 94 & $\begin{array}{l}0.15(0.03- \\
0.67)\end{array}$ & $\begin{array}{l}0.07(0.05- \\
0.16)\end{array}$ \\
\hline & $3 \mathrm{rd}(1.21-9.19)$ & 18.4 & 76 & $\begin{array}{l}0.11(0.03- \\
0.52)\end{array}$ & $\begin{array}{l}0.05(0.03- \\
0.06)\end{array}$ \\
\hline & $P$ trend & & & $P=0.019^{*}$ & $P=0.032$ \\
\hline \multirow[t]{4}{*}{ PFUnDA } & $1 \mathrm{st}(0.09-0.22)$ & 2.3 & 88 & 1 (reference) & $\begin{array}{l}1 \\
\text { (reference) }\end{array}$ \\
\hline & $\begin{array}{l}\text { 2nd(>0.22- } \\
0.39)\end{array}$ & 17.9 & 78 & $\begin{array}{l}0.11(0.02- \\
0.48)\end{array}$ & $\begin{array}{l}0.01(0.03- \\
0.75)\end{array}$ \\
\hline & $\begin{array}{l}3 r d(>0.39- \\
1.15)\end{array}$ & 16.7 & 84 & $\begin{array}{l}0.12(0.03- \\
0.53)\end{array}$ & $\begin{array}{l}0.07(0.01- \\
0.37)\end{array}$ \\
\hline & $P$ trend & & & $P=0.013^{*}$ & $P=0.007^{\star}$ \\
\hline \multirow[t]{4}{*}{ PFDA } & $1 \mathrm{st}(0.07-0.22)$ & 13.9 & 72 & 1 (reference) & $\begin{array}{l}1 \\
\text { (reference) }\end{array}$ \\
\hline & $\begin{array}{l}\text { 2nd }(>0.22- \\
0.3)\end{array}$ & 8.19 & 122 & $\begin{array}{l}1.81(0.71- \\
4.58)\end{array}$ & $\begin{array}{l}0.76(0.63- \\
1.84)\end{array}$ \\
\hline & $3 r d(0.33-0.65)$ & 17.9 & 56 & $\begin{array}{l}0.74(0.29- \\
1.93)\end{array}$ & $\begin{array}{l}0.69(0.56- \\
1.92)\end{array}$ \\
\hline & $P$ trend & & & $P=0.165$ & $P=0.435$ \\
\hline$\sum$ PFAS & 1st(2.8-6.85) & 19.7 & 61 & 1 (reference) & $\begin{array}{l}1 \\
\text { (reference) }\end{array}$ \\
\hline \multicolumn{6}{|c|}{ OR, odds ratio; $\mathrm{Cl}$, confidence interval } \\
\hline \multicolumn{6}{|c|}{ P-value for test of trend across tertiles } \\
\hline Adjusted for age(con & inuous),BMI(conti & uous) & & & \\
\hline
\end{tabular}




\begin{tabular}{|c|c|c|c|c|c|}
\hline Perfluorochemicals & Tertiles(ng/mL) & $\begin{array}{l}\text { cumulative live } \\
\text { birth per } \\
\text { aspiration (\%) }\end{array}$ & Cycles & $\begin{array}{l}\text { Crude } \\
\text { OR(95\%Cl) }\end{array}$ & $\begin{array}{l}\text { Adjusted } \\
\text { OR } \\
(95 \% \mathrm{Cl})\end{array}$ \\
\hline & $\begin{array}{l}\text { 2nd }(>6.85- \\
22.33)\end{array}$ & 5.4 & 111 & $\begin{array}{l}4.29(1.52- \\
12.09)\end{array}$ & $\begin{array}{l}1.76(1.43- \\
2.16)\end{array}$ \\
\hline & $\begin{array}{l}3 r d(>22.33- \\
38.08)\end{array}$ & 15.4 & 78 & $\begin{array}{l}1.35(0.59- \\
3.25)\end{array}$ & $\begin{array}{l}0.76(0.62- \\
1.93)\end{array}$ \\
\hline & $P$ trend & & & $P=0.019^{*}$ & $P=0.027^{*}$ \\
\hline \multicolumn{6}{|c|}{ OR, odds ratio; $\mathrm{Cl}$, confidence interval } \\
\hline \multicolumn{6}{|c|}{ P-value for test of trend across tertiles } \\
\hline \multicolumn{6}{|c|}{ Adjusted for age(continuous),BMI(continuous) } \\
\hline
\end{tabular}

Last, a reduced fertilization rate was found in the highest tertile PFHxS and $\sum$ PFAS groups (PFHxS $43.9 \%$ vs $68.9 \%, p \leq 0.001 ; \sum$ PFAS $52 \%$ vs $\left.74.2 \%, p \leq 0.001\right)$.

\section{Discussion}

Our results indicate that follicular fluid PFAS exposure was associated with increased odds of low probability of fertilization, clinical pregnancy and live birth among POR women. These findings are consistent with prior literature that has evaluated the relationship between PFASs and female fecundity[7, $8,9]$. The majority of studies have suggested that PFASs affect human fecundity through a longer time to pregnancy $(\mathrm{TTP})[7,8,9]$.

Unlike prior studies that followed a self-selected set of individuals who succeeded in conceiving, the research design in the present study focused on the impact of maternal PFAS exposure on ART outcomes. Our results provide evidence that elevated PFAS exposure reduced the probability of fertilization, pregnancy and live birth, which is partially in line with prior studies[16, 17]. For example, Governi $L$ observed a significant negative correlation $(R=0.75 ; p<0.001)$ between FF PFAS levels and the fertilization rate, and McCoy identified a negative relationship between FF PFDA and PFUnDA and blastocyst conversion rates[16, 17]. However, the researchers did not observe associations between PFAS exposure and pregnancy probability after $\operatorname{IVF}[16,17,18]$. This was largely due to sample size constraints given the relative infrequency with which such outcomes occurred (the maximum sample size was 38 ). It is also possible that women with poor ovarian response are sensitive to reproductive toxic substances.

The underlying mechanisms of PFAS-induced reproductive toxicity in humans remain largely unknown, although some in vitro studies have reported that PFAS can directly interact with oocytes and granulosa cells and influence oocyte quality and survival[11, 12,20]. For example, a recent study reported that $10 \mu \mathrm{g}$ $\mathrm{mL}^{-1}$ PFNA for $22 \mathrm{~h}$ had a severe negative effect on blastocyst formation in bovine oocytes in vitro[11], 
which could be attributed to the disturbance in lipid droplet distribution. Peroxisome proliferator-activated receptors (PPARs) are crucial for successful oocyte development and lipid metabolism [21, 22]. Previous experiments have shown that PFASs can bind and activate PPAR [23,24], and the activity of human PPAR-alpha increased with increasing carbon backbone chain length [25]. It is plausible that PFASs may interfere with ovarian cell function and oocyte maturation by interacting with PPARs.

Furthermore, PFAS exposure could affect oocyte survival by altering cell-cell communication within a follicle. When treated with PFDA during porcine oocyte maturation in vitro, gap junction intercellular communication (GJIC) among cumulus cells and oocytes was disrupted, and the number of live oocytes and the percentage of matured oocytes decreased[20]. Similarly, Domínguez A's study found that PFOS interfered with GJIC in cumulus-oocyte complexes during the first hours of oocyte maturation[12].

On the other hand, our results highlighted the declining trend of a well-known long-chain PFAS compound (PFOS) in FF. More specifically, the concentration of PFOS in our study was more than four times lower than that in previous studies that recruited patients in the period of 2006-2011 and in 2015[26, 27]. This finding confirms the decline in contamination with PFOS in China, which can be mainly attributed to the phasing out of PFOS-based products and the restriction of PFOS in industrial and consumer products by international organizations since 2009[28, 29]. In contrast to the PFOS ban, PFOA is classified as a highly concerning substance[30] but has not yet been completely phased out worldwide. This may be an explanation as to why our PFOA follicular concentrations were quite comparable with previous studies[16, 26, 27].

To our knowledge, we are the first to report that PFBA was the PFAS found at the highest concentration in FF $[16,26,27]$. The PFAS contamination pattern in FF is very similar to that in Chinese drinking water [31]: PFBA is detected at the highest concentration, followed by PFHpA, PFOA, and PFOS. There are two reasons for this phenomenon. First, the increase in PFASs in the surrounding environment would lead to an increase in PFASs in the human body. Drinking and dietary intake have been considered the main routes by which PFASs enter the human body [32]. Currently, short-chain PFASs, especially PFBA, have become the major PFAS contaminants in Chinese drinking water, wheat and vegetables [31, 33, 34, 35]. Evidence has also shown that ongoing exposure to even relatively low concentrations of PFASs in drinking water increases human serum levels [33]. Second, the blood-follicle transfer efficiencies for PFASs decrease with increasing chain length [34]. Although PFBA was the most abundant PFAS in FF, the present study failed to confirm any association between PFBA and ART outcomes. The possible reasons include the following: First, PFBA serum half-lives are much shorter than those of PFOA (48-96 h vs 1273 days) [36]. Second, short-chain PFBA showed less cytotoxicity, inhibition of aromatase activity and alteration of cellular lipids than long-chain homologs [37]. Since environmental long-chain PFASs have gradually decreased and been replaced by short-chain PFASs in China [31,35], the risks posed by shortchain PFASs require further investigation.

The present study has several limitations. First, our study focused on women with POR, and it may not be possible to generalize our findings to couples from the overall population of couples attempting 
conception. Second, we did not consider male partner exposure. Further studies are needed to explore whether maternal or paternal plasma PFASs had effects on IVF outcomes. The small sample size limited the interpretation of this study.

In conclusion, our study supports the association of higher FF PFAS contamination being associated with a lower chance of successful pregnancy and live birth, mainly due to a reduced fertilization rate. Additionally, this study confirmed that the levels of long-chain PFAS contamination are declining in the FF. The risks posed by short-chain PFASs require further investigation.

\section{Declarations}

\section{Ethics approval and consent to participate}

All experiments were performed in strict accordance with the Ethics Committee at The First People's Hospital of Yunnan Province, P.R. China. Informed consent was obtained from all subjects. The Institutional Committee of the First People's Hospital of Yunnan Province, P.R. China, approved the experimental protocols (registration number: 2017YY138).

\section{Consent for publication}

All coauthors have seen and approved the final version of the paper and have agreed to its submission for publication. All patients signed written informed consent forms.

\section{Availability of data and materials}

The datasets used and/or analyzed during the current study are available from the corresponding author upon reasonable request.

\section{Competing interests}

The authors declare that they have no competing interests.

\section{Funding}

The present study was supported by the Natural Science Foundation of China (Grant No. 81760143), the Foundation for High-Level Talents of Yunnan, China (Grant No. L-201624), "10,000 Talent Plan" of Yunnan Province (YNWR-MY-2019-020), and the Science and Technology Program of Yunnan, China (Grant No. U0120170150).

\section{Authors' contributions}

Patient selection: Y. C. and L. Z.; sample collection and processing: Y. C. and L. Z.; sample measurements: M. W.; data analysis and interpretation: Y. C. and L. Z.; study design: H. S. and Z. W.; manuscript drafting: Y. C. and H. S. All authors read and approved the final manuscript. 


\section{Acknowledgment}

The authors thank the staff of the Reproductive Medicine Center and Endocrinology Laboratory of The First People's Hospital of Yunnan Province, P.R. China, for sample collection and processing.

\section{References}

1. Ma X, Cui L, Chen L, Zhang J, Zhang X, Kang Q, Jin F, Ye Y. Parental plasma concentrations of perfluoroalkyl substances and In Vitro fertilization outcomes. Environ Pollut. 2021 Jan 15;269:116159. doi: 10.1016/j.envpol.2020.116159. Epub 2020 Nov 27. PMID: 33279270.

2. Centers for Disease Control. National Report on Human Exposure to Environmental Chemicals, 2019. https://www.cdc.gov/exposurereport/index.html

3. Ding N, Harlow SD, Randolph JF Jr, Loch-Caruso R, Park SK. Perfluoroalkyl and polyfluoroalkyl substances (PFAS) and their effects on the ovary. Hum Reprod Update. 2020 Sep 1;26(5):724-752.

4. Cardenas A, Hivert MF, Gold DR, Hauser R, Kleinman KP, Lin PD, Fleisch AF, Calafat AM, Ye X, Webster TF, Horton ES, Oken E. Associations of Perfluoroalkyl and Polyfluoroalkyl Substances With Incident Diabetes and Microvascular Disease. Diabetes Care. 2019 Sep;42(9):1824-1832. doi: 10.2337/dc182254. Epub 2019 Jul 11. PMID: 31296647

5. Wang J, Zeng XW, Bloom MS, Qian Z, Hinyard LJ, Belue R, Lin S, Wang SQ, Tian YP, Yang M, Chu C, Gurram N, Hu LW, Liu KK, Yang BY, Feng D, Liu RQ, Dong GH. Renal function and isomers of perfluorooctanoate (PFOA) and perfluorooctanesulfonate (PFOS): Isomers of C8 Health Project in China. Chemosphere. 2019 Mar;218:1042-1049.

6. Ye WL, Chen ZX, Xie YQ, Kong ML, Li QQ, Yu S, Chu C, Dong GH, Zeng XW. Associations between serum isomers of perfluoroalkyl acids and metabolic syndrome in adults: Isomers of C8 Health Project in China. Environ Res. 2021 May;196:110430.

7. Fei C, McLaughlin JK, Lipworth L, Olsen J. Maternal levels of perfluorinated chemicals and subfecundity. Hum Reprod. 2009;24(5):1200-1205. doi:10.1093/humrep/den490

8. Vélez MP, Arbuckle TE, Fraser WD. Maternal exposure to perfluorinated chemicals and reduced fecundity: the MIREC study. Hum Reprod. 2015 Mar;30(3):701-9.

9. Bach CC, Liew Z, Bech BH, et al. Perfluoroalkyl acids and time to pregnancy revisited: An update from the Danish National Birth Cohort. Environ Health. 2015;14:59. Published 2015 Jul 7. doi:10.1186/s12940-015-0040-9

10. Busnelli A, Papaleo E, Del Prato D, La Vecchia I, lachini E, Paffoni A, Candiani M, Somigliana E. A retrospective evaluation of prognosis and cost-effectiveness of IVF in poor responders according to the Bologna criteria. Hum Reprod. 2015 Feb;30(2):315-22.

11. Hallberg I, Kjellgren J, Persson S, Örn S, Sjunnesson Y. Perfluorononanoic acid (PFNA) alters lipid accumulation in bovine blastocysts after oocyte exposure during in vitro maturation. ReprodToxicol. 2019 Mar;84:1-8. 
12. Domínguez A, Salazar Z, Arenas E, Betancourt M, Ducolomb Y, González-Márquez H, Casas E, Teteltitla M, Bonilla E. Effect of perfluorooctane sulfonate on viability, maturation and gap junctional intercellular communication of porcine oocytes in vitro. Toxicol In Vitro. 2016 Sep;35:93-9

13. Hurley S, Goldberg D, Wang M, Park JS, Petreas M, Bernstein L, Anton-Culver H, Nelson DO, Reynolds P. Time Trends in Per- and Polyfluoroalkyl Substances (PFASs) in California Women: Declining Serum Levels, 2011-2015. Environ Sci Technol. 2018 Jan 2;52(1):277-287.

14. Su C, Lu Y, Wang T, Lu X, Song S, Li L, Khan K, Wang C, Liang R. Dynamic multimedia fate simulation of Perfluorooctane Sulfonate (PFOS) from 1981 to 2050 in the urbanizing Bohai Rim of China. Environ Pollut. 2018 Apr;235:235-244. doi: 10.1016

15. Meng J, Wang T, Song S, Wang P, Li Q, Zhou Y, Lu Y. Tracing perfluoroalkyl substances (PFASs) in soils along the urbanizing coastal area of Bohai and Yellow Seas, China. Environ Pollut. 2018 Jul;238:404-412.

16. McCoy JA, Bangma JT, Reiner JL, Bowden JA, Schnorr J, Slowey M, O'Leary T, Guillette LJ Jr, Parrott BB. Associations between perfluorinated alkyl acids in blood and ovarian follicular fluid and ovarian function in women undergoing assisted reproductive treatment. Sci Total Environ. 2017 Dec 15;605606:9-17.

17. Governini L, Orvieto R, Guerranti C, Gambera L, De Leo V, Piomboni P. The impact of environmental exposure to perfluorinated compounds on oocyte fertilization capacity. J Assist Reprod Genet. 2011 May;28(5):415-8.

18. Petro EML, D'Hollander W, Covaci A, Bervoets L, Fransen E, De Neubourg D, De Pauw I, Leroy JLMR, Jorssen EPA, Bols PEJ. Perfluoroalkyl acid contamination of follicular fluid and its consequence for in vitro oocyte developmental competence. Sci Total Environ. 2014 Oct 15;496:282-288.

19. Ma X, Cui L, Chen L, Zhang J, Zhang X, Kang Q, Jin F, Ye Y. Parental plasma concentrations of perfluoroalkyl substances and In Vitro fertilization outcomes. Environ Pollut. 2021 Jan 15;269:116159. doi: 10.1016/j.envpol.2020.116159. Epub 2020 Nov 27. PMID: 33279270.

20. Domínguez A, Salazar Z, Betancourt M, Ducolomb Y, Casas E, Fernández F, Bahena I, Salomón A, Teteltitla M, Martínez R, Chaparro A, Cuapio P, Salazar-López C, Bonilla E. Effect of perfluorodecanoic acid on pig oocyte viability, intracellular calcium levels and gap junction intercellular communication during oocyte maturation in vitro. Toxicol In Vitro. 2019 Aug;58:224-229.

21. Dupont J, Chabrolle C, Ramé C, Tosca L, Coyral-Castel S. Role of the peroxisome proliferatoractivated receptors, adenosine monophosphate-activated kinase, and adiponectin in the ovary. PPAR Res. 2008;2008:176275.

22. Froment P, Gizard F, Defever D, Staels B, Dupont J, Monget P. Peroxisome proliferator-activated receptors in reproductive tissues: from gametogenesis to parturition. J Endocrinol. 2006 May;189(2):199-209.

23. Das KP, Grey BE, Rosen MB, Wood CR, Tatum-Gibbs KR, Zehr RD, Strynar MJ, Lindstrom AB, Lau C. Developmental toxicity of perfluorononanoic acid in mice. ReprodToxicol. 2015 Jan; 51:133-44. 
24. DeWitt JC, Shnyra A, Badr MZ, Loveless SE, Hoban D, Frame SR, Cunard R, Anderson SE, Meade BJ, Peden-Adams MM, Luebke RW, Luster MI. Immunotoxicity of perfluorooctanoic acid and perfluorooctane sulfonate and the role of peroxisome proliferator-activated receptor alpha. Crit Rev Toxicol. 2009;39(1):76-94.

25. Wolf CJ, Takacs ML, Schmid JE, Lau C, Abbott BD. Activation of mouse and human peroxisome proliferator-activated receptor alpha by perfluoroalkyl acids of different functional groups and chain lengths. Toxicol Sci. 2008 Nov;106(1):162-71.

26. Heffernan AL, Cunningham TK, Drage DS, Aylward LL, Thompson K, Vijayasarathy S, Mueller JF, Atkin SL, Sathyapalan T. Perfluorinated alkyl acids in the serum and follicular fluid of UK women with and without polycystic ovarian syndrome undergoing fertility treatment and associations with hormonal and metabolic parameters. Int J Hyg Environ Health. 2018 Aug;221(7):1068-1075.

27. Kim YR, White N, Bräunig J, Vijayasarathy S, Mueller JF, Knox CL, Harden FA, Pacella R, Toms LL. Perand poly-fluoroalkyl substances (PFASs) in follicular fluid from women experiencing infertility in Australia. Environ Res. 2020 Nov;190:109963.

28. Zhou Z, Liang Y, Shi Y, Xu L, Cai Y. Occurrence and transport of perfluoroalkyl acids (PFAAs), including short-chain PFAAs in Tangxun Lake, China. Environ Sci Technol. 2013 Aug 20;47(16):924957.

29. UNEP, 2009. The conference of the parties 4 of the Stockholm Convention (COP-4) in Geneva placed perfluorooctane sulfonate and perfluorooctane sulfonyl fluoride(PFOS and PFOSF) in Annex B. http://chm.pops.int/Convention/Pressrelease/COP4Geneva9May2009/tabid/542/language/enUS/Default.aspx

30. USEPA, 2013. The 2010/2015 PFOA stewardship program. http://www.epa.gov/oppt/pfoa/pubs/stewardship/, Accessed date: 19 November 2015

31. Li Y, Li J, Zhang L, Huang Z, Liu Y, Wu N, He J, Zhang Z, Zhang Y, Niu Z. Perfluoroalkyl acids in drinking water of China in 2017: Distribution characteristics, influencing factors and potential risks. Environ Int. 2019 Feb;123:87-95.

32. Jian JM, Guo Y, Zeng L, Liang-Ying L, Lu X, Wang F, Zeng EY. Global distribution of perfluorochemicals (PFCs) in potential human exposure source-A review. Environ Int. 2017 Nov;108:51-62.

33. Liu Z, Lu Y, Shi Y, Wang P, Jones K, Sweetman AJ, Johnson AC, Zhang M, Zhou Y, Lu X, Su C, Sarvajayakesavaluc $S$, Khan K. Crop bioaccumulation and human exposure of perfluoroalkyl acids through multi-media transport from a mega fluorochemical industrial park, China. Environ Int. 2017 Sep;106:37-47.

34. Kang Q, Gao F, Zhang X, Wang L, Liu J, Fu M, Zhang S, Wan Y, Shen H, Hu J. Nontargeted identification of per- and polyfluoroalkyl substances in human follicular fluid and their blood-follicle transfer. Environ Int. 2020 Jun;139:105686.

35. Scher DP, Kelly JE, Huset CA, Barry KM, Hoffbeck RW, Yingling VL, Messing RB. Occurrence of perfluoroalkyl substances (PFAS) in garden produce at homes with a history of PFAS-contaminated 
drinking water. Chemosphere. 2018 Apr;196:548-555.

36. Chang SC, Das K, Ehresman DJ, Ellefson ME, Gorman GS, Hart JA, Noker PE, Tan YM, Lieder PH, Lau C, Olsen GW, Butenhoff JL. Comparative pharmacokinetics of perfluorobutyrate in rats, mice, monkeys, and humans and relevance to human exposure via drinking water. Toxicol Sci. 2008 Jul;104(1):40-53.

37. Gorrochategui E, Pérez-Albaladejo E, Casas J, Lacorte S, Porte C. Perfluorinated chemicals: differential toxicity, inhibition of aromatase activity and alteration of cellular lipids in human placental cells. Toxicol Appl Pharmacol. 2014 Jun 1;277(2):124-30. 